\title{
Filigrane
}

Écoutes psychanalytiques

\section{Prométhée se déchaîne. Entre savoir technologique et pouvoir de mort}

\section{Ghyslain Lévy}

Volume 25, numéro 1, printemps 2016

Actes du colloque Le sujet sacrifié (Ghyslain Lévy)

URI : https://id.erudit.org/iderudit/1037370ar

DOI : https://doi.org/10.7202/1037370ar

Aller au sommaire du numéro

Éditeur(s)

Revue Santé mentale au Québec

ISSN

1192-1412 (imprimé)

1911-4656 (numérique)

Découvrir la revue

Citer cet article

Lévy, G. (2016). Prométhée se déchaîne. Entre savoir technologique et pouvoir de mort. Filigrane, 25(1), 19-32. https://doi.org/10.7202/1037370ar d'utilisation que vous pouvez consulter en ligne. 


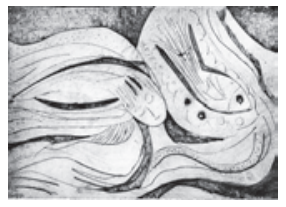

\title{
Prométhée se déchaîne. Entre savoir technologique et pouvoir de mort
}

\author{
Ghyslain Lévy
}

Il est malheureusement évident qu'aujourd'hui la technologie a dépassé l'humanité.

Albert Einstein

Nous ne savons pas comment tirer le sens de cette horreur. Nous n'en sommes pas capable. Car il est impossible de l'appliquer à notre expérience humaine ou à notre temps humain... Alors vaut-il mieux se souvenir ou oublier? Svetlana Alexievitch, La supplication

a première question que j'aimerais partager avec vous concernerait le — champ de la psychanalyse comme participant à l'intelligibilié et à la mise en sens de la réalité, tenant compte d'un reste qui lui serait irréductible. Ou pour le dire autrement, dans un monde que le sens a abandonné, à quelle place situer le lieu du champ analytique?

Pour parler de cette violence d'expulsion du sens, j'ai choisi aujourd'hui de me confronter à ce qui, du réel, subsiste comme un reste non subjectivable, un reste particulièrement actif sous forme d'une voix qui ordonne la réalité: je veux parler de la voix de la technique, et en sa pointe extrême, la voix du technonucléaire, ce «maître qui veille à la mort», pour reprendre le titre d'un livre de René Major (1992). Le technonucléaire est le reste d'une opération où la folie de la rationalité érigée en savoir absolu, irréfutable, est venue rejoindre aujourd'hui les tendances destructrices les plus archaïques et les plus primitives de l'humain.

Renforcée par la croyance passionnelle dans une figure quasi religieuse du Progrès, la voix de la technique est venue s'imposer dans tous les appareils de pouvoir, et s'étendre jusque dans les psychés individuelles, comme la voix qui commande au consentement meurtrier. Elle rejoint ici la voix d'une pulsion de pouvoir qui trouve collectivement et individuellement, en ses points de fascination, l'occasion de réinvestir les traces d'une cruauté originaire sans culpabilité. Le langage du technonucléaire intime aujourd'hui l'ordre 
de l'indifférence envers ce qui est fait en termes de destruction et d'altération, et c'est sous cet impératif implicite qu'il prétend à sa supposée insignifiance. «Dans l'effondrement de toute expérience éthique qui définit notre temps, écrit Giorgio Agamben, “je me donne l'ordre d'obéir”.» (Agamben, 2014, p. 58) Ne serait-ce pas là, la dernière injonction paradoxale d'un impératif technique de mort, et qui fait de l'intériorisation de la voix du commandement, le fondement de la fabrication de «l'homme nouveau»? Cette nouvelle chimère ne serait-elle pas la seule désormais à pouvoir se soumettre à l'emprise technique de la destruction? On l'appelle aujourd'hui l'humanisme technologique.

On pourrait poser la question autrement: dans un monde où il n'y a plus de scène sur laquelle pouvoir jouer le théâtre de la cruauté, quel sens la psychanalyse peut-elle avoir?

Avant de déployer ces questions, une petite remarque préalable: il y a un siècle exactement, en mars 1915, Freud écrivait, au milieu du carnage qui ensanglantait l'Europe, un texte sur la guerre et sur notre indifférence envers la mort. Sa lecture, en 2015, est d'une actualité troublante. Car c'est d'une profonde désillusion dont nous parle Freud. La désillusion de celui qui avait accordé une si grande importance à la communauté de culture, au progrès et à l'esprit des Lumières, à ce que Freud appelait la délicatesse éthique envers l'autre. «Et voilà que la guerre à laquelle nous ne voulions pas croire, fit irruption et apporta la désillusion.» (Freud, 1915, p. 13) Une guerre sauvage, brutale, qui va retourner avec cynisme le progrès et sa surenchère technique dans le sens du meurtre de masse, et lancer les masses elles-mêmes avec passion les unes contre les autres, sans plus aucune limitation morale, et pour la plus grande satisfaction des pulsions égoïstes déchaînées.

Je suis frappé par la lucidité de Freud qui, en 1915, s'interroge sur cette passion du meurtre qui saisit l'homme de la culture, les peuples dits civilisés, les nations soumises aux valeurs morales les plus hautes. C'est notre relation inconsciente à la mort qui a changé, la mort de l'autre, du plus proche, du plus aimé, au plus étranger, au plus lointain, qui se révèle en fin de compte nous laisser dans une indifférence qui peut aller jusqu'à la satisfaction de sa disparition. Quant à notre propre mort, elle ne rencontre aussi en nous qu'une sorte d'indifférence puisque nous n'y croyons pas. Notre angoisse de mort n'est que quelque chose de secondaire, issue le plus souvent d'une conscience de culpabilité.

Je trouve remarquable l'effort que s'impose Freud, en 1915, pour penser l'irreprésentable de cette folie de masse où l'effroi se mêle aux passions 
sanguinaires, dans le même déni de la mort et dans l'indifférence au sort de l'autre. Ce qui amène Freud à cette conclusion: si nous devons en juger selon nos souhaits inconscients et la sorte d'insensibilité qui en est la conséquence, nous sommes bien tous, comme les hommes originaires, une bande de meurtriers. Ne sommes-nous pas dominés, chaque jour, à chaque heure, par cette sorte d'indifférence envers l'autre qui s'exprime dans le désir inconscient de l'envoyer «au diable», c'est-à-dire à la mort, quand le Narcisse souverain se sent endommagé en nous?

Il y a un siècle, face à l'hécatombe qui saignait l'Europe comme jamais encore, la psychanalyse avec Freud cherchait à penser ce qui jusqu'à présent demeure encore soigneusement réprimé: la nécessité d'éclairer notre rapport inconscient à la mort, de lever le voile de déni quant à notre destin mortel, afin de «nous organiser en vue de la mort» condition pour continuer à endurer la vie.

Ce que Freud relève en 1915, confronté à l'horreur de la guerre et au déchaînement d'une emprise des techniques de mort, me semble de la plus haute importance. Car c'est pour lui de désillusion dont il s'agit. Or si l'illusion est d'abord le fait du jeu, du rêve mais aussi du dispositif théâtral, du praticable sur lequel il s'agit de jouer le drame de la vie, le cadre qui rend possible la représentation de la réalité, son nécessaire fictionnement, l'illusion demeure aussi la condition même pour penser et rêver la scène psychique sur laquelle le sujet pourra transposer les enjeux inconscients de désir à l'épreuve de sa réalité. Toute l'expérience de la psychanalyse consiste à construire ces dispositifs rendant possible de rêver la réalité, de la déréaliser et donc de s'en dé-fasciner en l'absentant en quelque sorte. C'est ce que j'ai appelé le don de l'ombre.

Mais avec la guerre, Freud découvre que le voile de l'illusion nécessaire pour penser la réalité, mais aussi pour la jouer, que ce voile s'est déchiré. La désillusion est le dévoilement de cette déchirure, l'effondrement de tout praticable sur lequel transférer la réalité et sa rencontre avec les motions de souhaits inconscients. Plus de déplacement possible de la réalité sur une autre scène qui l'irréaliserait en la rêvant. Face à la folie sanguinaire du monde, à sa destructivité, la désillusion freudienne dévoile l'excès de visibilité du réel et son emprise sur la pensée qu'il paralyse.

\section{Une réalité indifférente}

Me confrontant au texte de Freud, son actualité me trouble quand je constate combien, à ce tableau de la guerre technique, tel que Freud en 
trace les grands traits en 1915, s'ajoute aujourd'hui la chape d'indifférence envers la précarité d'un monde soumis à la menace froide d'une technoscience nucléaire capable de vitrifier des populations entières à distance et sans haine. Plus question d'un face à face entre des combattants lancés dans des affrontements sanglants terrifiants, sur lesquels Freud s'interrogeait. Ma lecture du texte de 1915 me renvoie à un étrange sentiment de vide, de perte d'humanité à l'instant où, pensant à la déshumanisation de la guerre, je perçois ce qu'il y a d'insaisissable dans notre insensibilité aujourd'hui, mais peut-être aussi dans notre incrédulité, dans notre consentement passif, détaché, insouciant, plein de bonne conscience humaniste envers la cruauté désaffectée de notre technoscience de mort.

Alors quelle place aujourd'hui pour la psychanalyse, ou plutôt quelle scène? Peut-être que cette interrogation trouvera peu à peu, tout au long de ce texte, un certain nombre de réponses. Mais elle m'amène à avancer déjà sur un terrain qui m'importe au plus haut point. Car face aux désastres humains, environnementaux et écologiques présents et à venir, conséquences de l'explosion des réacteurs nucléaires des Centrales de Tchernobyl et de Fukushima devenues des bombes nucléaires civiles, nous restons hébétés, apathiques ou pire encore, indifférents, comme non concernés. Nous sommes dans la désillusion freudienne.

Or ce sont là des manifestations psychiques et émotionnelles qui surviennent quand il s'agit de faire face à un événement traumatique inattendu, qui a débordé notre capacité de penser et de ressentir ou même d'imaginer ce qui se passe. C'est précisément là une expérience qui se rencontre dans le champ de la psychanalyse des situations extrêmes. Par ce débordement traumatique de la psyché, l'événement ne peut y inscrire ses traces mnésiques. C'est comme si, du point de vue de notre ressenti intérieur, il n'avait pas de réalité psychique, il n'était pas encore arrivé. Nous n'en percevons encore rien, nous n'en avons aucune expérience. Car pour que la réalité extérieure puisse exister du point de vue du sujet, elle doit «être traduite en psychique» (Aulagnier, 1975). C'est tout le travail de la perlaboration dans la cure. Mais cette traduction, cette transformation en matière psychique ne se fait que sur fond «d'une indifférence objective du réel», comme l'écrit Nathalie Zaltzman (1998).

Nous raisonnons ainsi: «Je sais bien que les catastrophes nucléaires ont eu lieu, je sais bien que cela peut encore avoir lieu à tout moment, mais quand même je n'y crois pas.» Nous restons incrédules. Cette apathie anesthésiée est aujourd'hui la chose la plus partagée au regard des menaces réelles 
que constituent les Centrales nucléaires qu'une technologie affolée multiplie dans la plus totale irresponsabilité, et dont le pouvoir de mort se déchaîne, dénoué de toute capacité de penser et d'anticiper le risque. C'est pourquoi je demeure convaincu que, comme ce fut nécessaire pour Freud en 1915, la psychanalyse aujourd'hui se doit de répondre de sa propre expérience, quand la relation au monde est elle-même devenue une situation globale en totale perte de sens, et quand, sous l'effet d'une technologisation généralisée du rapport à l'autre, c'est le pouvoir de mort qui se déchaîne, dénoué de tout lien de pensée et d'affect.

\section{Prométhée, une nouvelle figure du mythe}

Günther Anders qui a été le penseur le plus engagé dans une réflexion courageuse sur le feu nucléaire et ses conséquences, a lui aussi noté l'importance de cette réaction apathique chez les survivants d'Hiroshima. Il écrit: «Lorsque j'ai cherché à parler de la déflagration atomique avec les victimes d'Hiroshima, elles restaient tout simplement muettes [...] L'événement était trop grand pour qu'ils aient pu s'en rappeler et même pour qu'ils aient pu le percevoir... Les victimes ne pouvaient plus se rappeler ce qu'on leur avait fait.» (Anders, 2007, p. 72-73)

Une telle escalade technologique de mort, je l'ai nommée «le déchaînement de Prométhée» dont la version soft, énergétique, est représentée par le commerce des Centrales nucléaires aujourd'hui. Prométhée, cette figure du mythe qui a dérobé le feu divin et qui depuis, enchaîné à un rocher, se fait sans cesse dévorer le foie par un aigle, Prométhée le voleur de feu a, depuis Hiroshima et Nagasaki, rompu ses chaînes. Libéré de ce qui faisait son entrave ontologique à un destin limité et mortel, et détaché de ses liens par l'accès à une technologie capable de menacer désormais l'humanité de l'homme, Prométhée se déchaîne, ou du moins faut-il l'entendre comme l'extension au plan collectif du déchaînement dans le moi des pulsions destructrices désentravées. Freud l'avait déjà souligné dès 1920, la destructivité pulsionnelle se déchaîne quand rien ne vient plus la lier à un amour de soi et à un amour de l'autre sous l'égide des pulsions sexuelles.

Prométhée déchaîné représente désormais la figure même d'une toute-puissance dont l'homme a toujours rêvé, mais un rêve qui se réalise aujourd'hui dramatiquement avec le développement exponentiel d'une technologie nucléaire affolée. Celle-ci ne tenant plus compte de ce qui devrait la retenir, la brider par la réalité, ce que figure le rocher de Prométhée, ne connaît désormais que la jouissance du pire, avec cette multiplication des 
bombes civiles potentielles que constituent les centrales nucléaires à très haut risque. Le Prométhée antique n'est-il pas désormais enchaîné non plus à son rocher mais à son double gémellaire, Epiméthée, celui qui, littéralement, "ne pensait qu'après»? Autrement dit Prométhée déchaîné ne renvoie-t-il pas à cette autre figure du mythe, un Epiméthée inconséquent qui agit sans penser ou qui agit avant de penser aux conséquences de ses actes?

Epiméthée serait la figure d'une technique qui aujourd'hui devient folle, et s'affolant, se précipite dans la catastrophe annoncée. Le mythe se déchaîne désormais dans le réel, par une progression exponentielle des technologies les plus irresponsables, sans que nous puissions nous représenter les conséquences de ce que nous fabriquons. Désormais Prométhée se déchaîne dans l'éhonté de son désir grandiose, un Prométhée qui ne saurait plus «penser avant d'agir », mais pris dans le vertige du pire à venir.

Donc Prométhée se déchaîne. Cet intitulé à ma communication aujourd'hui s'est imposé au moment où j'ai pris connaissance d'une décision qui me semble encore si ahurissante qu'elle ne peut susciter que l'incrédulité: comment croire qu'un contrat ait pu être passé récemment entre la France et le Japon pour la construction d'une centrale nucléaire en Turquie, plus précisément dans la région de Sinop, au nord de la Turquie, sur le bord de la Mer Noire, dans une des zones sismiques les plus dangereuses de la région? En 1999, c'était à Izmit, dans la grande banlieue d'Istanbul, qu'un séisme avait causé plus de 20000 morts. Cette centrale franco-japonaise sera dotée de six réacteurs de la dernière génération, à partir d'un contrat géant de 16,5 milliards d'euros. La conséquence immédiate enregistrée à la Bourse de Paris a été alors le bond des actions AREVA à $+2,8 \%$ et celles de EDF à $+1,4 \%$. Depuis lors, on sait l'effondrement des entreprises du nucléaire en France, en raison des engagements financiers «délirants» sur des projets similaires témoignant des fantasmes de toute-puissance de leurs dirigeants.

Participant à cette euphorie financière internationale, les propos du premier ministre japonais, Shinz Abe, ont quelque chose qui fait frémir : «Il est de la responsabilité du Japon de partager son expérience et les leçons qu'il a tirées de l'accident de Fukushima et de contribuer ainsi à l'amélioration de la sécurité nucléaire du monde.» Que M. Abe place ainsi la participation actuelle de son pays à la construction de nouvelles centrales nucléaires dans le monde sous le signe de la responsabilité, ce qui va de pair avec la remise en activité des centrales japonaises stoppées depuis Fukushima, a de quoi surprendre. Il en serait donc, selon le premier ministre japonais, de la responsabilité du Japon que de partager son savoir-faire nucléaire à la suite 
d'une catastrophe (appelée ici «accident») qui est loin d'être terminée et contrôlée à la centrale de Fukushima dai-ichi. Car nous sommes toujours dans Fukushima. C'est cela qu'il est important de comprendre et qui est précisément l'objet du déni.

C'est d'ailleurs sur le même thème de la responsabilité que répondent les arguments des responsables turcs: «Notre pays est dans une zone sismique dangereuse, mais nous mettons en œuvre des mesures pour sécuriser les sites que nous construisons.» Comment faire tenir ensemble ces deux assertions, l'une qui est un constat, celui d'une réalité sismique dangereuse qui devrait écarter radicalement la seconde assertion elle-même et la promesse de sécurité? Il y a une violence dans l'absurdité et les contradictions des dirigeants politiques qui, au nom du principe de responsabilité, portent atteinte à la cohérence même de la réalité. Comment des régimes démocratiques aujourd'hui, dont celui de la France, et sans l'ombre de la moindre réserve, peuvent-ils prendre en commun la décision de construire un site nucléaire sur des failles sismiques reconnues par tous, et en des lieux de récentes catastrophes telluriques, alors même que les conséquences humaines et environnementales du désastre de Fukushima sont loin d'avoir trouvé un début de résolution? Au nom de quel cynisme les responsables turcs peuvent-ils déclarer, quelques jours après l'événement de Fukushima: «Il n'y a pas d'investissement sans risque. Nous comptons maintenant les mois, voire les semaines, avant d'entamer avec la Russie notre projet de centrale nucléaire à Akkuyu.»?

«Il n'y a pas d'investissement sans risque»... C'est à la lettre le principe auquel le monde de la finance se règle pour justifier sa course infinie vers plus de profits par des opérations toujours plus risquées et irresponsables. Or, avec les bombes civiles que constituent les centrales nucléaires, tout se passe comme si les décideurs politiques et économiques oubliaient qu'ils avaient à faire non à des flux financiers abstraits mais avec la réalité humaine et son environnement vivant. D'ailleurs le «comme si» est ici de trop. La violence qui, dans ces différentes sphères, traite de l'humain comme de la matière inerte à évaluer quantitativement, est bien la réalité de notre temps.

Comment le principe de responsabilité qui est régulièrement invoqué comme alibi moral par les décideurs peut-il être soutenu dans un tel déni de la réalité humaine et de la peur collective que ce déni suscite? Quand la réalité humaine est traitée comme une stricte réalité monétaire, l'occasion d'investissements visant le plus grand profit et la plus forte rentabilité, tenant compte d'une part de risque quant à la perte sur investissement, c'est 
que le consentement qui est alors exigé des hommes relève d'un système sacrificiel, ou plutôt autosacrificiel. Cette question est aujourd'hui d'autant plus cruciale que l'État nucléaire qui est la nouvelle forme de nos démocraties technologiquement avancées est aussi celui qui ne cesse de construire une nouvelle langue pour s'adresser à ses concitoyens; une nouvelle langue qui doit sans cesse être démasquée quand elle détourne le sens des mots comme ceux de responsabilité, de sécurité, de risque, d'investissement, mais aussi de dissuasion, ou de savoir-faire... Une novlangue pour un usage pervers et inversé de leur sens.

On en conclura ici que l'existence de ces bombes civiles potentielles, ces centrales nucléaires construites aujourd'hui sur des sites à haute fréquence sismique, démontrent la cruauté de nos systèmes démocratiques quand ils s'alignent sur les intérêts d'une finance mondiale la plus sauvage et la plus incontrôlée. N'oublions pas d'autre part que toute politique nucléaire qui s'avance sous le masque «tranquille» du souci énergétique ne fait qu'occulter les perspectives militaro-industrielles qui se trament en arrière-plan.

\section{Le principe responsabilité et l'écosystème humain}

Avec Hiroshima et Nagasaki, on pourrait dire que la critique de la technique introduite par Heidegger a pris une dimension angoissante universelle puisque c'est la relation de l'homme à son humanité qui est aujourd'hui sous condition. Autrement dit, c'est à une réhabilitation de la notion de responsabilité que nous devons conduire notre réflexion commune, un terme si perverti aujourd'hui, comme je viens de le montrer, dans les discours des dirigeants de nos démocraties. Et c'est bien sûr à l'autre penseur de cette situation, Hans Jonas, que je dois ici faire appel; Hans Jonas qui, en 1992, soutenait déjà la nécessité «d'un principe responsabilité» au regard de la menace nucléaire globale. Cette menace globale est notre réalité quotidienne puisque nous la vivons à travers la multiplication des Fukushimas à venir. D'où la question: comment notre vie psychique s'organise-t-elle au regard d'une réalité du monde placée sous une telle menace?

Plus généralement, ce principe responsabilité doit être mesuré à l'aune de la menace qu'une technoscience déshumanisée fait peser sur l'ensemble humain à considérer comme faisant partie de ce que j'appellerais l'écosystème vivant général. C'est là un saut épistémique fondamental: on peut dire que la conception classique de l'homme, seul à faire face aux forces déchaînées de la nature, en conflit avec elle pour sa survie, se révèle désormais obsolète. Avec le franchissement inédit que représente le largage des 
premières bombes atomiques sur les populations civiles de grands centres urbains, avec la démultiplication des centrales nucléaires à très hauts risques, c'est l'ensemble des écosystèmes de la planète qui sont menacés d'extinction. Et c'est le rapport ontologique de l'homme dans la nature qu'il est alors essentiel de repenser.

Le sujet contemporain, pour sa survie, doit absolument se penser comme faisant partie des écosystèmes naturels que sa technique met en danger. Il en est donc de la responsabilité de chacun, en tant que sujet et en tant que citoyen, de se considérer lui-même comme un des écosystèmes de son environnement. C'est là la condition éthique de sa survie: se penser individuellement et collectivement comme un écosystème humain en danger. C'est là un axiome fondamental pour une éthique du futur. Ces conditions fragiles de l'existence de l'écosystème humain, chacun se doit d'en répondre aujourd'hui, pour son propre compte mais aussi pour le compte de l'autre, à entendre aussi pour le compte des générations à venir, puisque la durée de contamination par les substances radioactives se mesure au moins par centaines d'années.

Prométhée se déchaîne, il se confond désormais avec son jumeau Epiméthée, incapable d'anticiper les effets catastrophiques de ses actes. Abolir le rapport à la nature dans l'humain règle radicalement toute la relation qu'entretient l'écosystème humain avec les autres écosystèmes de son environnement. Sous l'alibi d'améliorer la condition humaine présente en préparant la mutation post-humaine, à partir des biotechnologies, nanotechnologies, intelligence artificielle et de l'information, c'est la vision machinique du monde qui l'emporte, fondée sur les valeurs de transparence, de maîtrise et de contrôle visant à fabriquer « un homme» que la nature aurait fait déficitaire sinon déficient. Or la gestion des centrales nucléaires ne se revendique-t-elle pas, elle aussi, d'une technologie transparente, totalement maîtrisée et donc entièrement sous contrôle, même si, comme les catastrophes récentes l'ont prouvé à l'envi, la réalité est toute autre? Ce sont les mêmes valeurs qui sont communes à la situation nucléaire contemporaine et à cette idéologie de «l'homme nouveau», technologiquement fabriqué.

N'y a-t-il pas bien souvent pour le sujet, ce dont témoigne l'ère techniciste, un refus et même une honte de son origine sexuée, honte de n'être qu'un sujet limité par sa condition mortelle, mais honte aussi d'avoir été engendré dans une rencontre de sujets désirants, d'être né d'une conception qu'il n'a pas choisie, n'a pas maîtrisée, et dans laquelle il n'a pas eu son mot à dire? N'y a t-il pas chez le sujet une honte de ne rien pouvoir changer à 
ce destin d'engendrement qui l'entame dans sa liberté toute-puissante de Narcisse, une honte d'avoir un corps qui le limite, et une génétique qui le contraint? Avec Freud et la psychanalyse, s'est aussi ajoutée la honte de ne plus être maitre chez soi puisque des forces inconscientes de désirs nous dominent et nous déterminent. À cette crise narcissique du sujet cherche à répondre le développement des technologies du post-humain dont font partie les manipulations génétiques du vivant.

À l'âge d'une vision machinique du monde, la réalité technonucléaire occupe une place prépondérante et indiscutée, par la remise en question de l'existence de l'homme comme espèce et de son environnement vital, mais aussi en apportant une étrange «réponse» par ce qu'on appelle aujourd'hui le post-humanisme. On pourrait aussi l'appeler «le temps de la post-catastrophe», selon Thierry Hoquet qui écrit dans son livre Cyborg philosophie: «Les individus amputés [mais de quel désastre!] et réparés par prothèse robotique sont en réalité le laboratoire où s'expérimentent les formes de vie de l'avenir.» (Zarka, 2013, p. 3) L'humanisme post-humain serait comme la tentative de penser une suite au désastre de l'humain que prépare un avenir nucléaire. Après l'amputation de l'humain, «l'humain sera prolongé, c'està-dire à la fois amplifié et potentiellement annihilé par l'avènement d'autre chose qui n'est pas lui.»

Ici le développement exponentiel d'une technologie nucléaire dévastatrice de l'écosystème humain, et d'autre part l'extension du domaine du post-humain se rejoignent dans le même refus du rapport ontologique de l'homme à la nature. Comme l'écrit Yves Charles Zarka: «il faut abolir tout ce qui reste de naturel dans l'homme pour que celui-ci puisse s'accomplir dans un être autre qui ne connaîtrait plus la naissance, la maladie, l'échec, la souffrance et la mort... [...] avènement d'un être déchargé des limites que lui impose son corps précaire, fragile, insatisfait et mortel.» (Zarka, 2013, p. 3-4). L'on voit bien la fonction fétiche qu'occupe l'objet technique dans la vie psychique confrontée aux angoisses de castration et aux limites du narcissisme. Seul un tel être post-humain pourrait consentir à l'obsolescence de l'homme destiné à l'avenir nucléaire et à une telle désillusion.

\section{Un devoir d'angoisse}

Tel est l'enjeu d'une vision techniciste du monde qui fait du savoir technique un nouveau pouvoir de mort. Au regard de cet enjeu, Anders nous rappelle à notre devoir d'angoisse (Anders, 2007, p. 109). Seul un devoir d'angoisse peut construire une éthique du futur donnant encore une place 
à l'humanité de l'homme. Parler de devoir d'angoisse peut paraître paradoxal, mais c'est précisément un tel paradoxe qui se révèle riche de sens, car c'est à partir de l'angoisse comme le signal en nous du surgissement d'une menace, d'un danger, d'une urgence traumatique, que Anders nous rappelle à une position de sujet. Autrement dit l'angoisse serait comme un signal d'alerte qui se manifeste quand une réalité destructrice s'impose menaçant notre survie, alors même que tout en nous cherche à annuler cette réalité. La position de sujet que rappelle Anders réside précisément dans le fait de ne pas consentir à nier en soi l'existence d'émotions comme l'angoisse, la peur, mais aussi la haine que les situations catastrophiques de Fukushima, de Tchernobyl, les destructions historiques des villes nipponnes peuvent susciter en nous.

A contrario, je pense à un récent documentaire vu à la télévision sur les suites désastreuses de l'explosion nucléaire, à Fukushima. Un de ses habitants déclarait: "J'en veux à TEPCO comme beaucoup de gens ici, ce serait un mensonge que de ne pas le reconnaître. Mais je suis fonctionnaire, et je ne suis donc pas en position de ressentir de la haine.» Beaucoup de choses sont dites là quant au clivage mental imposé à chacun pour nous contraindre à cette violence sur soi qui consiste à devoir consentir à une science nucléaire qui joue à l'apprenti sorcier et à une société qui, sous l'alibi du progrès et de l'opulence, en vient à menacer de mort l'existence de l'humain.

Or c'est en acceptant de reconnaître en soi l'angoisse et la haine, que pourra s'exprimer le refus individuel et collectif à un tel consentement. Refuser de consentir au sacrifice de l'avenir serait notre devoir d'angoisse, celui de regarder le risque en face. C'est contre l'indifférence molle envers cette réalité, ou contre le clivage de la réalité que je signalais précédemment, que le principe éthique nous rappelle au devoir d'être angoissé. C'est la leçon que nous laisse Anders, au regard d'une situation nucléaire dans laquelle cynisme, avidité au gain, cruauté et indifférence conduisent au malheur. À ce devoir d'angoisse il faudrait ajouter le travail psychique nécessaire à chaque sujet et qui consiste à reconnaître la participation de notre destructivité pulsionnelle à ce consentement meurtrier.

Ici et avec son principe du devoir d'angoisse, Anders rejoint Jonas avec son principe responsabilité. Deux penseurs de la fin du siècle dont les œuvres respectives ont été, je le rappelle, d'abord et définitivement marquées par l'horreur d'Auschwitz et par cette production industrielle de millions de cadavres, comme ce fut le cas dans les camps de mort nazis. C'est probablement en ayant en tête cette monstruosité qu'ils ont assisté avec effroi à la 
fabrication d'une masse vitrifiée en un éclair atomique, comme ce fut le cas à Hiroshima puis à Nagasaki.

Mort éclair ou mort au ralenti. Cette situation se caractérise chaque fois par un état de sidération de la pensée chez ceux qui ont essayé de se représenter la catastrophe. C'est ce que confie Anders: "Après Hiroshima, je suis resté muet, non parce que je n'avais pas saisi la monstruosité de l'événement, mais parce qu'au contraire, mon imagination ma pensée, ma bouche et ma peau, tout cela refusait de travailler devant la monstruosité de l'événement.» (Anders, 2007, p. 64). Svetlana Alexievitch (2004) va même plus loin dans son livre La supplication à propos des rescapés de l'après-Tchernobyl quand elle écrit: "Chaque chose reçoit son nom lorsqu'elle est nommée pour la première fois. Il s'est produit un événement pour lequel nous n'avons ni système de représentation, ni analogies, ni expérience. Un événement auquel ne sont adaptés ni nos yeux, ni nos oreilles, ni même notre vocabulaire [...] Une nouvelle histoire des sens vient de commencer...» (p. 59).

"Le mythe de demain» est une gigantesque fresque qui a été conçue pour le métro de Tokyo, afin d'exposer les effets du désastre de Fukushima. Des milliers de personnes passent devant la fresque chaque jour, mais personne n'y prête attention, chacun passe comme s'il n'y avait rien à voir. Tel serait le tracé du non-événement ou plutôt l'exposition de son image qui est à la lettre invisible, parce que placée dans un excès de visibilité. Car la fresque du métro de Tokyo ne fonctionne pas manifestement comme une scène sur laquelle aurait lieu la représentation du désastre, son ombre. Les scènes de la représentation ont quitté le monde. C'est peut-être cela la véritable violence, quand il n'y a plus d'ombre qui puisse abriter la représentation du réel, produire la scène fictionnelle de la catastrophe, son invisibilité.

Le monde a perdu sa ou ses scènes sur lesquelles il pouvait jouer son irréalité. Il est devenu technique, comme l'image elle-même. La scène a désormais laissé la place à l'écran, mais à un écran qui ne fait plus écran, qui ne cache plus rien, qui ne fait plus médiation entre l'image du réel et le réel de l'image (Mondzain, 2015). Ou, pour le dire autrement, le réel, sous la souveraineté de la voix de la technique, ne se reproduit pas, ne se représente pas, mais se répète. La fresque du métro de Tokyo n'est qu'un écran sur lequel la voix techno-nucléaire de TEPCO continue à ordonner ce qui est bon et mauvais pour la communauté, en répétant en boucle ininterrompue l'image obturante et donc insignifiante d'un nom, Fukushima.

«Le visible tisse une toile compacte, où le vide et la distance sont absorbés par le marché des choses propres à combler tous les manques», écrit 
Marie-José Mondzain (2015, p. 66). À quoi j'ajouterais que parmi ces choses comblantes, le flux d'images, dans son excès de présence et de visibilité, vient produire une tache aveugle qui s'étend dans la psyché de chacun et diffuse de proche en proche. De même un tel flux de visibilité vient créer une zone d'anesthésie de plus en plus vaste. Personne ne voit rien car il n'y a rien à voir, ni rien à sentir. Le scotome de la réalité psychique a effacé la perception d'une partie du monde extérieur.

Exhiber la sidération du regard serait peut-être la seule façon de pouvoir témoigner d'un tel scotome et d'un processus extensif qui diffuse à tout le reste de la réalité perceptive. Le scotome s'étend aussi à la langue elle-même car ce sont les mots qui, en perdant toute relation à la réalité, ont perdu tout sens. Il n'y a plus de mots pour dire certaines choses. Cet écrasement des mots, cette récusation de la possibilité d'habiter les mots est directement la conséquence de cette violence du visible. Avec les mots, c'est aussi la récusation des affects, l'impossibilité d'habiter les éprouvés que suscitent la réalité. C'est là une question fondamentale pour la psychanalyse aujourd'hui à la recherche de sa propre scène.

En 1958, Anders se rendit à Hiroshima et à Nagasaki. Il note dans son Journal: «La constance que les Japonais mettent à ne pas parler des coupables, à taire que l'événement a été causé par des hommes, à ne pas nourrir le moindre ressentiment, bien qu'ils aient été les victimes du plus grand des crimes, c'en est trop pour moi, cela dépasse l'entendement.» (Dupuy, 2009, p. 252-253). Et il ajoute: «Le monde offre l'image d'un paradis habité par des meurtriers sans méchanceté et par des victimes sans haine.» On pourrait préciser: des meurtriers amnésiques devenus «les amis» imposés à leurs victimes sans haine. "Cette absence de haine sera l'absence de haine la plus inhumaine qui ait jamais existé, écrit Marc Crépon, absence de haine et absence de scrupule ne feront plus qu'un.» (Crépon, 2012, p. 312) C'est précisément ce que j'entends comme le primat psychique d'un principe d'indifférence.

La haine de même que la peur ou l'angoisse seraient-elles devenues superflues? Notre croyance en la toute-puissance de la technique nous aurait-elle transformés en individus techniques, dont tous ces sentiments, peut-être trop humains, seraient devenus obsolètes? Un principe d'indifférence ne devient-il pas souverain? Quant à la folie fanatique contemporaine, ne réalise-t-elle pas le retour sanglant d'un refoulé de la haine, de la peur, de l'angoisse, que nos sociétés occidentales sophistiquées n'ont eu de cesse d'occulter et ce, jusqu'à aujourd'hui? 
Retrouver en partage de tels éprouvés, retrouver par le travail psychique, celui de la psychanalyse, de l'art, et en général de toutes les œuvres de pensée, la possibilité de construire une scène sur laquelle jouer le théâtre d'ombre de la réalité, n'est-ce pas la condition pour faire ensemble communauté de partage de la réalité humaine elle-même? Non pas une communauté dont l'idéal d'unité serait enfin réalisé à partir des dénis de la réalité, mais une communauté qui puisse se reconnaître dans le souci de chacun, regardant de biais les brisures du monde, de se dé-fasciner de ses éclats médusants, de son excès de visibilité. Ne s'agirait-il pas de retrouver le chemin d'une certaine illusion nécessaire contre la désillusion imposée par le réel tel que Freud en faisait l'amer constat en 1915 dans ses Considérations actuelles sur la guerre et la mort?

\author{
Ghyslain Lévy \\ ghyslainlevy@orange.fr
}

\title{
Références
}

Anders, G. (2007). Et si je suis désespéré, que voulez-vous que j'y fasse? Paris: Allia.

Agamben, G. (2013). Qu'est-ce que le commandement? Paris: Payot rivages.

Alexievitch, S. (2004). La supplication. Tchernobyl, chronique du monde après l'apocalypse (extraits). Diogène, 207 (3), 59-63.

Aulagnier, P. (1975). La violence de l'interprétation. Paris: Presses universitaires de France. Crépon, M. (2012). Le consentement meurtrier. Paris: Édition du Cerf.

Freud, S. (1915). Considérations actuelles sur la guerre et la mort. Dans Essais de psychanalyse. France: Petite Bibliothèque Payot, 1995, 9-40.

Jonas, H. (1998). Pour une éthique du futur. Paris: Payot rivages.

Levy, G. (2015). Le don de l'ombre. Paris: Campagne Première.

Major, R. (1992). Des idéaux en partage: ou comment se donner un maître qui veille à la mort?

Dans L'éthique du don. Jacques Derrida et la pensée du don. Paris: Métaillé-Transition.

Mondzain, M.-J. (2015). L'image peut-elle tuer? Paris: Bayard.

Zaltzman, N. (1998). La réalité est-elle paranoïaque? Topique, 67, 37-56.

Zarka, Y.C. (2013). Aujourd'hui le post-humain. Cités, 3 (55), 3-8. 